\title{
Data quantity is more important than its spatial bias for predictive species distribution modelling
}

\author{
Willson Gaul ${ }^{\text {Corresp., } 1}$, Dinara Sadykova ${ }^{2}$, Hannah J White ${ }^{1}$, Lupe Leon-Sanchez ${ }^{2}$, Paul Caplat ${ }^{2}$, Mark C Emmerson ${ }^{2}$, \\ Jon M Yearsley ${ }^{1}$ \\ ${ }^{1}$ School of Biology and Environmental Science, University College Dublin, Dublin, Ireland \\ 2 School of Biological Sciences, The Queen's University Belfast, Belfast, United Kingdom \\ Corresponding Author: Willson Gaul \\ Email address: willson.gaul@ucdconnect.ie
}

Biological records are often the data of choice for training predictive species distribution models (SDMs), but spatial sampling bias is pervasive in biological records data at multiple spatial scales and is thought to impair the performance of SDMs. We simulated presences and absences of virtual species as well as the process of recording these species to evaluate the effect on species distribution model prediction performance of 1) spatial bias in training data, 2) sample size (the average number of observations per species), and 3) the choice of species distribution modelling method. Our approach is novel in quantifying and applying real-world spatial sampling biases to simulated data. Spatial bias in training data decreased species distribution model prediction performance, but sample size and the choice of modelling method were more important than spatial bias in determining the prediction performance of species distribution models. 


\section{Data quantity is more important than its spatial bias for predictive}

\section{species distribution modelling}

Willson Gaul ${ }^{1 *}$, Dinara Sadykova ${ }^{2}$, Hannah J. White ${ }^{1}$, Lupe León-Sánchez ${ }^{2}$, Paul Caplat ${ }^{2}$, Mark

C. Emmerson ${ }^{2}$, Jon M. Yearsley ${ }^{1}$

${ }^{1}$ School of Biology and Environmental Science, University College Dublin, Dublin, Ireland

${ }^{2}$ School of Biological Sciences, The Queen's University Belfast, Belfast, United Kingdom

Corresponding Author:

Willson Gaul ${ }^{1}$

Email address: willson.gaul@ucdconnect.ie 


\section{ABSTRACT}

2 Biological records are often the data of choice for training predictive species distribution

3 models (SDMs), but spatial sampling bias is pervasive in biological records data at multiple

4 spatial scales and is thought to impair the performance of SDMs. We simulated presences and

5 absences of virtual species as well as the process of recording these species to evaluate the effect

6 on species distribution model prediction performance of 1) spatial bias in training data, 2) sample

7 size (the average number of observations per species), and 3) the choice of species distribution

8 modelling method. Our approach is novel in quantifying and applying real-world spatial

9 sampling biases to simulated data. Spatial bias in training data decreased species distribution

10 model prediction performance, but sample size and the choice of modelling method were more

11 important than spatial bias in determining the prediction performance of species distribution

12 models.

\section{INTRODUCTION}

15 Biological records data ("what, where, when" records of species identity, location, and date of

16 observation) often contain large amounts of data about species occurrences over large spatial

17 areas (Isaac \& Pocock, 2015). Knowing the geographic areas occupied by species is important

18 for practical and fundamental research in a variety of disciplines. Epidemiologists use maps of

19 predicted wildlife distributions to identify areas at high risk for wildlife-human transmission

20 (Deka \& Morshed, 2018; Redding et al., 2019). Land managers can use knowledge of species

21 distributions in spatial planning to minimize impacts on wildlife of new infrastructure (Dyer et

22 al. 2017; Newson et al., 2017). Because complete population censuses are not available for most 
23 species, species distribution models (SDMs) are often used to predict distributions of species

24 using relatively sparse observations of species. Species observation data used to train SDMs

25 must represent the study area, but when studies focus on scales of thousands (or tens- or

26 hundreds of thousands) of square kilometers, it is difficult and often expensive to collect

27 adequate data across the entire study extent. Spatially random or stratified sampling of species

28 across large spatial areas is possible, and such surveys exist for some taxa including butterflies

29 and birds (Uzarski et al., 2017), but such data are uncommon for most taxonomic groups (Isaac,

30 van Strien, August, de Zeeuw, \& Roy, 2014). More commonly, data are either spatially extensive

31 but collected opportunistically (Amano, Lamming, \& Sutherland, 2016), or are collected

32 according to structured study designs but are more spatially limited.

33 Collecting biological records data is relatively cheap compared to collecting data directly as

34 part of a research project (or at least the costs of collecting biological records are borne in large

35 part by individual observers rather than by data analysts) (Carvell et al., 2016). However, there is

36 an associated challenge because the analyst lacks control over where, when, and how data were

37 collected. Many biases have been documented in biological records data, including temporal,

38 spatial, and taxonomic biases (Boakes et al., 2010). Spatial sampling bias, in which some areas

39 are sampled preferentially, is particularly pervasive at all scales and across taxonomic groups

40 (Amano \& Sutherland, 2013; Oliveira et al., 2016). Despite these biases, biological records are

41 often used in species distribution models. Given the ubiquitous presence of spatial sampling bias

42 in biological records data, it is important to know whether spatial bias in training data impedes

43 the ability of SDMs to correctly model species distributions. Data collection efforts often face a

44 practical trade-off between maximizing the overall quantity and the spatial evenness of new

45 records. It would thus be useful to know whether the value of biological records for SDMs can 
46 best be improved by increasing the spatial evenness of recording (perhaps at the cost of the

47 overall amount of new data that is added), or by increasing the overall amount of recording (even

48 if new records are spatially biased).

49 Spatial sampling bias in biological records has similarities with sampling biases that have been

50 investigated in other settings. The field of econometrics uses the term "sample selection bias" to

51 refer to non-random sampling and has developed theory about when sampling bias is likely to

52 bias analyses (Wooldridge, 2009). A key consideration in econometrics' evaluations of sample

53 selection bias is determining whether the inclusion of data in the sample depends on predictor

54 variables that are included in the model ("exogenous" sample selection), or depends on the value

55 of the response variable ("endogenous" sample selection), or both (Wooldridge, 2009). In

56 ecology, Nakagawa (2015) similarly provides guidelines for assessing missing data in terms of

57 whether data is missing randomly or systematically with respect to other variables (see also

58 Gelman \& Hill, 2006). In a machine learning context, Fan, Davidson, Zadrozny, \& Yu (2005)

59 investigated the effect on predictive models of sample selection bias in which sampling is

60 associated with predictor variables - "exogenous sample selection" in the terms of Wooldridge

61 (2009) and "missing at random" in the terms of Nakagawa (2015) - and determined that most

62 predictive models could be sensitive or insensitive to sampling bias depending on particular

63 details of the dataset.

64 Biological records may have been collected with spatial sampling biases that are exogenous,

65 endogenous, or both, and datasets may contain a mix of records collected with different types of

66 bias. For example, when sampling intensity depends on proximity to roads (Oliveira et al., 2016),

67 the sampling bias is exogenous because records arise from biased sampling that depends on an

68 aspect of environmental space that can be included in models as a predictor variable. However, 
69 when a birder, for example, submits a record of an unusual bird from a location where they

70 would not otherwise have submitted records, the bias is endogenous because the sampling

71 location depends on the value of the response variable (species presence). Most sampling biases

72 occur on a continuum and are not unequivocally categorizable using any existing scheme

73 (Nakagawa, 2015), making it difficult to describe exactly the biases in data or predict their effect

74 on model performance.

75 Studies testing the impact of spatially biased training data on predictive SDMs have shown

76 mixed results (Edwards, Cutler, Zimmerman, Geiser, \& Moisen, 2006; Phillips et al., 2009;

77 Barbet-Massin, Jiguet, Albert, \& Thuiller, 2012; Stolar \& Nielsen, 2015). Phillips et al. (2009)

78 found that spatial bias in the presence records strongly reduced model performance when using a

79 pseudo-absence approach but not when using a presence-absence approach. Using a virtual

80 ecologist simulation approach (Zurell et al., 2010), Thibaud, Petitpierre, Broennimann, Davison,

$81 \&$ Guisan (2014) found that the effect of spatial sampling bias on SDM prediction performance

82 depended on the SDM modelling method, and that the effect of spatial sampling bias was smaller

83 than the effect of other factors, including sample size and choice of modelling method. Warton,

84 Renner, \& Ramp (2013) provided a method for correcting for spatially biased data when building

85 SDMs, but found that the resulting improvement in model predictive performance was small.

86 Because there is no clear guidance about when spatial bias in training data will or will not affect

87 model predictions, tests of the observed effect of spatial biases common in biological records are

88 important for determining whether those biases are likely to be problematic in practice.

89 The effect of spatial sampling bias on model predictions can be studied using real or simulated

90 data (Zurell et al., 2010; Meynard, Leroy \& Kaplan, 2019). Using real data has the advantage

91 that the biases in the data are, well, real. The spatial pattern, intensity, and correlation of 
92 sampling bias with environmental space are exactly of the type that analyses of real data must

93 cope with. However, using real data has two disadvantages. First, the truth about the outcome

94 being modeled (species presence or absence) is not completely known in the real world, making

95 it impossible to evaluate how well models represent the truth. Second, biases in real data are not

96 limited to the biases under study - a study investigating the effect of exogenous spatial sampling

97 bias will be unable to exclude from a real dataset records generated by endogenously biased

98 sampling that depends on the values of the outcome variable. Simulation studies avoid both these

99 problems. Because the investigator specifies the underlying pattern that is subsequently modeled,

100 the truth is known exactly (even when realized instances of the simulation are generated with

101 some stochasticity). The investigator also has direct control over which biases are introduced into

102 a simulated dataset, and therefore can be more confident that any observed effects on predictions

103 are due to the biases under investigation.

104 Spatial sampling bias can be introduced into simulated data using a parametric function that 105 describes the bias (Isaac et al., 2014; Stolar \& Nielsen, 2015; Thibaud et al., 2014; Simmonds,

106 Jarvis, Henrys, Isaac \& O'Hara, 2020) or by following a simplified ad-hoc rule (e.g. splitting the

107 study region into distinct areas that are sampled with different intensities) (Phillips et al., 2009).

108 However, these approaches may not adequately test the effect of spatial bias if the biases found

109 in real biological records do not follow parametric functions or are more severe than artificial

110 parametric or ad-hoc biases. We used observed sampling patterns from Irish biological records to

111 sample simulated species distributions using realistic spatially biased sampling.

112 We used a virtual ecologist approach (Zurell et al., 2010; Meynard, Leroy \& Kaplan, 2019)

113 applied at the scale of Ireland to investigate the effect on the predictive performance of SDMs of

114 1) spatial sampling bias, 2) sample size (the average number of records per species), and 3) 
115 choice of SDM method. We quantified the spatial sampling biases used in our study to enable

116 comparison with biases in other datasets. To the best of our knowledge, our approach is novel in

117 applying real-world spatial sampling biases, derived directly from spatial sampling patterns in

118 existing datasets, to simulated virtual species.

\section{METHODS}

120 We assessed the ability of species distribution models to predict "virtual species" distributions

121 (Leroy, Meynard, Bellard, \& Courchamp, 2016; Zurell et al., 2010) when the models were

122 trained with datasets with a range of spatial sampling biases and sample sizes. The simulation

123 and analysis process is illustrated in Fig. 1. Virtual species distributions were produced by

124 defining the responses of virtual species to environmental predictor variables (Table 1, section

125 2.1) using the approach described in section 2.4.1. We then created maps of "true" virtual

126 species distributions covering $84010 \mathrm{~km}$ x $10 \mathrm{~km}$ grid squares in Ireland (total area of study

127 extent $=84,000 \mathrm{~km}^{2}$ ). We then generated "virtual biological records" by sampling presence-

128 only records from the community of virtual species in each grid square, using sampling patterns

129 taken from real Irish biological records data. We then inferred non-detections for each species

130 using presence records of other species (van Strien, van Swaay, \& Termaat, 2013). SDMs were

131 trained using the environmental variables as predictors and the virtual species detection/non-

132 detection data as the response. Model prediction performance was evaluated using three

133 measures of prediction performance (see section 2.5).

\section{$134 \quad 2.1 \quad$ Environmental predictor variables}

135 We chose environmental predictor variables with a range of spatial patterns and scales of

136 spatial auto-correlation (Table 1, Fig. S1). We used real environmental variables measured over 
137 a real geographic space, so the variety of spatial patterns in our predictor variables should be

138 similar to patterns in variables that determine biological species distributions at this scale, adding

139 realism to our simulation. We used climate variables (which show relatively strong spatial

140 clustering, Table 1) from the E-OBS European Climate Assessment and Dataset EU project

141 (Haylock et al., 2008; van den Besselaar, Haylock, van der Schrier, \& Klein Tank, 2011). We

142 calculated the proportion of each grid square covered by different land cover variables (which

143 show less spatial clustering than climate variables, Table 1) from the CORINE Land Cover

144 database (CORINE, 2012). We calculated the average elevation within each grid square by

145 interpolation using ordinary kriging from the ETOPO1 Global Relief Model (Amante \& Eakins, 146 2009).

147 Spatial data were prepared using the 'sf', 'sp', 'raster', 'fasterize', 'rgdal', 'gstat', and

148 'tidyverse' packages in R version 3.6 (Bivand, Keitt, \& Rowlingson, 2018; Gräler, Pebesma, \&

149 Heuvelink, 2016; Hijmans 2018; Pebesma, 2018; R Core Team, 2020; Ross, 2018; Wickham, 150 2017).

\section{$\begin{array}{lll}151 & 2.2 & \text { Species presence data }\end{array}$}

152 We downloaded observations of species across the island of Ireland for the years 1970 to 2014

153 from the British Bryological Society for bryophytes and from the Irish National Biodiversity

154 Data Centre (NBDC) for moths, butterflies, and birds. We used data for taxa that varied in both

155 their popularity with recorders and the ease with which species can be identified, because we

156 expected this would translate into different spatial sampling biases in the data. The data

157 contained presence-only records of species, with the date and location of the observation, and an 158 anonymized observer identifier. Locations of records were provided as either $1 \mathrm{~km}^{2}$ or $100 \mathrm{~km}^{2}$

$159(10 \mathrm{~km} \times 10 \mathrm{~km})$ grid squares, but we used $10 \mathrm{~km} \times 10 \mathrm{~km}$ grid squares in all analyses in order to 
160 retain the majority of the data. Within each taxonomic group, we grouped records into sampling

161 event checklists, where a sampling event was defined as all records with an identical

162 combination of recording date, location, and observer.

\section{$163 \quad$ 2.3 Spatial sampling patterns in Irish species presence data}

164 For each taxonomic group, we quantified sampling effort in each grid square as the proportion

165 of all records coming from the grid square. We quantified spatial sampling bias by calculating

166 the spatial evenness of sampling effort among locations using Simpson evenness (Magurran \&

167 McGill, 2011).

\section{$168 \quad 2.4 \quad$ Data simulation}

\section{2.4.1 Simulating species distributions}

170 We simulated and sampled virtual species distributions using the 'virtualspecies' package

171 (Leroy et al., 2016) in R. For each virtual species, seven environmental variables (Table 1) were

172 randomly chosen to use as drivers of occurrence (only seven of the ten variables shown in Table

1731 were used for each species so that not all species responded to exactly the same environmental

174 variables). The seven selected environmental variables were centered, scaled, and summarized

175 using principal components analysis with the 'ade4' R package (Dray \& Dufour, 2007). The

176 probability of occurrence of each virtual species $i$ in each grid square $j$ was a logistic function of

177 the first two principal components and their quadratic terms:

$178 \quad \operatorname{logit}\left(p_{i j}\right)=\alpha_{i}+\sum_{k=1}^{2}\left(\beta_{1 k i} V_{k j}+\beta_{2 k i} V_{k j}^{2}\right)$

179 where $p_{i j}$ is the probability that virtual species $i$ occurs in grid square $j, V_{k j}$ indicates the value of

180 the $k^{\text {th }}$ principal component in grid cell $j$, and the $\alpha$ and $\beta$ terms are the species-specific 
181 coefficients defining the response of the virtual species to the environment. The coefficients for

182 each virtual species $\left(\alpha, \beta_{1 k}, \beta_{2 k}\right)$ were chosen to ensure that each virtual species was present in

183 at least eight of the 840 grid squares.

\section{$184 \quad 2.4 .2 \quad$ Realized species distributions}

185 A single realized distribution of each virtual species $i$ was created by randomly generating a

186 "presence" (1) or "absence" (0) for each grid square $j$ by drawing a value from a binomial

187 distribution with probability $p_{i j}$. We simulated a community containing 1268 virtual species (the

188 number of recorded bryophyte species in Ireland). For comparison, results of a simulation using

189 a small community of 34 species are in Supplementary Article S1).

\section{$190 \quad$ 2.4.3 Sampling realized species with spatial bias}

191 Virtual biological records data were generated by sampling the realized species communities in

192 "sampling events" at different locations to produce spatially explicit species checklists (Fig. S2).

193 Spatial sampling locations were chosen based on spatial sampling patterns from three Irish

194 biological records datasets with different spatial sampling biases (Table 2): birds (low spatial

195 sampling bias), butterflies (median spatial sampling bias), and moths (severe spatial sampling

196 bias). This gave four spatial sampling "templates", including the case of no spatial sampling bias

197 (Fig. 2).

198 To make sampling patterns comparable between datasets with different sample sizes, we

199 calculated a relative sampling weight for each grid square in each empirical dataset (where the

200 most heavily sampled cell had a weight of one) by counting the number of records in each grid

201 square and dividing by the maximum number of records in any grid square (Fig. 2). 
202 We created virtual biological records with six different sample sizes, defined as the mean

203 number of records per species (number of records per species $=2,5,10,50,100$, and 200).

204 To generate virtual biological records from the virtual species communities, we randomly

205 selected a grid square, using selection probabilities from one of the four spatial-bias templates.

206 Within each grid square that was selected for sampling, we 1) generated a list of virtual species

207 that were present in the grid square; 2) defined the probability of observing each of the present

208 species based on the species' prevalence in the entire study extent (so that common species had a

209 higher probability of being recorded when present), and 3) drew presence observations with

210 replacement from the list of present species. We continued this sampling process until we had

211 accumulated the desired number of records.

\section{$212 \quad 2.5 \quad$ Species distribution modeling}

213 We tested three different SDM modeling techniques: generalized linear models (GLMs)

214 (Hosmer \& Lemeshow, 2000), boosted regression trees (Elith, Leathwick, \& Hastie, 2008;

215 Friedman, 2001), and inverse distance-weighted interpolation (Cressie, 1991). These represent

216 distinct types of methods used for SDMs, including linear and machine learning methods, and a

217 spatial interpolation method that does not include information from environmental covariates.

218 For all methods, the modeled quantity was the probability of the focal virtual species being

219 recorded on a checklist. We modeled each species individually as a function of five

220 environmental predictor variables, chosen from the ten possible predictor variables listed in

221 Table 1. Using only five of the ten possible predictor variables simulated a real-world situation in

222 which the factors that influence species distributions are not entirely known. We treated the list

223 of records from each sampling event as a complete record of that sampling event, and treated the

224 absence of species from a sampling event checklist as non-detection data for those species (Fig. 
225 S2, van Strien, van Swaay, \& Termaat, 2013; Kéry et al., 2010). Thus, we explicitly used a

226 detection/non-detection rather than a presence-only modeling framework. Our approach of

227 inferring non-detections of species at the locations of presence records of other species in the

228 community is similar to the "target-group background" approach of Phillips et al. (2009). Using

229 non-detection data inferred from records of other species ensured that the sampling biases were

230 the same for detections and non-detections, which may reduce the effect of sampling bias

231 (Barbet-Massin et al. 2012, Johnston et al. 2020, Phillips et al. 2009).

232 We modeled 110 randomly selected virtual species from the 1268 virtual species in the large

233 community simulation. The number of virtual species modeled was a compromise between high

234 replication and computation limitations, but testing 110 virtual species should provide enough

235 replication for robust conclusions. We fitted each type of SDM once to each combination of

236 virtual species, sample size, and spatial sampling bias. Thus, the sample size for our study - the

237 number of SDM prediction performance values that we used to assess the effects of spatial

238 sampling bias, sample size, and SDM method - was 110 prediction performance values for each

239 combination of SDM method, sample size, and spatial sampling bias. Replication in our study

240 came not from repeatedly fitting models to different randomly generated sets of presences and

241 absences of the same virtual species, but rather from fitting each model once to data for many

242 different virtual species, all generated using parameters randomly drawn from the same

243 distributions. However, the same 110 virtual species were used for each combination of SDM

244 method, spatial sampling bias, and sample size, ensuring that all comparisons were based on the

245 same underlying task (i.e. modelling the same true species distributions).

246 Models were trained and evaluated using five-fold spatial block cross-validation (Roberts et al.,

247 2017) that partitioned the study extent into spatial blocks of $100 \mathrm{~km} \times 100 \mathrm{~km}$ and allocated each 
248 block to one of five cross-validation partitions. We only attempted to fit models if there were

249 more than five presence records in the training data. Prediction performance of models was

250 evaluated using the true virtual species presence or absence in each grid cell not included in the

251 spatial extent of the training partitions (Fig. 3). Thus, evaluation data was spatially even and the

252 number of evaluation points stayed constant even as the sample size and spatial bias of training

253 data changed (Fig. 3). Prediction performance was evaluated using three metrics. The area

254 under the receiver operating characteristic curve (AUC) (Hosmer \& Lemeshow, 2000) evaluated

255 models' ability to accurately rank locations where species were present or absent using the

256 continuous SDM predictions, Cohen's Kappa (Cohen 1960), calculated using the threshold that

257 maximised Kappa, evaluated models ability to transform continuous SDM predictions into

258 binary maps of presence and absence, and root mean squared error (RMSE) evaluated model

259 calibration.

260 To train GLM SDMs, we used logistic regression ('glm' function) with a binomial error

261 distribution and logit link. Quadratic terms were fitted, but we did not fit interactions between

262 variables. We controlled overfitting by limiting the number of terms in GLMs such that there

263 were at least 10 detections or non-detections (whichever was smaller) in the training data for

264 each non-intercept term in the model. If a quadratic term was included in a model, we also

265 included the $1^{\text {st }}$ degree term. For generating predictions, we used the model that gave the lowest

266 AIC based on the training data.

267 Boosted regression trees were trained using 'gbm.step' in the 'dismo' package (Greenwell,

268 Boehmke, \& Cunningham, 2018; Hijmans, Phillips, Leathwick, \& Elith, 2017). Unlike GLMs,

269 boosted regression trees do not require the modeler to specify interactions between variables,

270 because the trees will discover and model interactions if they are present. The tree complexity 
271 specified by the modeler controls the maximum interaction order that the models are permitted to

272 fit, and therefore can be used to prevent overfitting. We tested tree complexities of two and five,

273 to build models that allowed interactions between up to two and up to five variables,

274 respectively. We used learning rates small enough to grow at least 1000 trees (following Elith,

275 Leathwick, and Hastie 2008), but large enough to keep models below an upper limit of 30,000

276 trees because of computation time limitations. We used gbm.step to determine the optimal

277 number of trees for each model, based on monitoring the change in 10-fold cross-validated error

278 rate as trees were added to the model (Hijmans, Phillips, Leathwick, \& Elith, 2017). Limiting

279 models to a maximum of 30,000 trees did not affect our results. Details of the procedure are in

280 Supplementary Article S1 and in our R code (see Data and code accessibility statement).

281 Inverse distance-weighted interpolation was implemented using 'gstat' (Gräler et al., 2016;

282 Pebesma, 2004). We tuned parameters of the inverse distance-weighted interpolation model

283 based on prediction error (details in Supplementary Article S1).

284 After models were fitted, we looked for evidence of overfitting and assessed whether the

285 number of presence records of the focal species in the test dataset affected prediction

286 performance metrics (details in Supplementary Article S1). All analyses used R version 3.6.0 (R

287 Core Team, 2020).

\section{$288 \quad 2.6 \quad$ Analyzing effects of sampling bias and sample size}

289 We modeled the predictive performance (AUC, maximum Cohen's Kappa, and RMSE) of

290 SDMs as a function of spatial sampling bias, sample size (average number of observations per

291 species), and SDM method. Modelling was done using boosted regression trees ('gbm.step' in

292 the 'dismo' package) (Greenwell et al., 2018; Hijmans et al., 2017). To assess whether species 
293 prevalence (the commonness or rarity of a species in the study extent) affected our evaluations of

294 model performance, we graphed prediction performance as a function of species prevalence for

295 all models (Fig. S3). We included species prevalence in the boosted regression tree models of

296 RMSE, but not in our analysis of AUC or Cohen's Kappa, because only RMSE showed a strong

297 trend with species prevalence (Fig. S3). Variable importance was assessed based on the

298 reduction in squared error attributed to each variable in boosted regression tree models

299 (Friedman, 2001). We also assessed the effect of spatial sampling bias and sample size of

300 training data on the number of species for which models could be fitted within the computational

301 time and memory constraints of this study (Supplementary Article S1).

\section{RESULTS}

303 Simulated species showed a variety of plausible distribution patterns (Fig. 4) and prevalences

304 (Fig. S4), including species with north/south distribution gradients and distributions that

305 followed geographic features such as the coastline (Fig. 4).

306 Sample size (the mean number of observations per species) and choice of SDM method were

307 the most important variables for explaining variations in prediction performance of SDMs (Table

308 3). Spatial sampling bias was the least important variable for explaining variation in prediction

309 performance for all three performance metrics (Table 3). Simpson evenness values for spatial

310 sampling evenness of the template datasets are in Table 2.

\section{$311 \quad 3.1 \quad$ Number of species successfully modeled}

312 The number of species for which models fitted successfully increased as sample size increased

313 and spatial bias decreased (Fig. 5). For GLMs and inverse distance-weighted interpolation,

314 model fitting was largely successful when datasets had more than 100 records per species, except 
315 when spatial bias was severe (Fig. 5). Boosted regression trees failed to fit models for some

316 species even with relatively large amounts of data (e.g. an average 200 records per species), and

317 models fit less frequently when data had median or severe spatial biases (Fig. 5). The effect of

318 spatial bias on the number of species for which models fitted was small, but was slightly greater

319 for boosted regression trees than for other SDM modelling methods (Fig. 5).

\subsection{Predictive performance of SDMs}

321 The amount of spatial bias in training data was less important than sample size and choice of 322 SDM method in predicting the performance of SDMs (Table 3, Table S1). AUC for predictive

323 SDMs increased with the average number of records per species and with decreasing spatial bias

324 in the training data when using all SDM methods (Fig. 6, Fig. 7). Root mean squared error

325 (RMSE) was largely unaffected by spatial sampling bias (Fig. 8, Table 3). Cohen's Kappa

326 differed between SDM methods, but generally increased with sample size, and with decreasing

327 spatial bias in the training data (Fig. S5, Fig. S6). However, Cohen's Kappa was low for all

328 methods and combinations of sample size and spatial bias when evaluated using spatial block

329 cross-validation (Fig. S6). The low Kappa scores indicated that our models were unable to

330 generalize beyond the training data when the task was to produce binary maps, despite the fact

331 that the models retained the ability to generalize when the task was to rank sites according to the

332 continuous SDM outputs (Supplementary Article S1, Fig. S7). Species prevalence (the number

333 of grid squares occupied by a species) had a negligible effect on the average value of AUC,

334 though it did affect the variability of AUC (Fig. S3). Species prevalence strongly affected the

335 expected value of RMSE, with RMSE increasing with species prevalence (Table 3, Fig. S3). 
336

337

338

339

340

341

342

343

344

345

346

347

348

349

350

351

352

353

354

355

356

\subsubsection{Effect of sample size}

Sample size (average number of records per species) was the most important variable for

predicting species distribution model prediction performance in terms of AUC (Table 3). AUC improved with increasing average number of records per species for all SDM methods, and the improvement in AUC decelerated as the number of records per species increased (Fig. 6, Fig. 9). Kappa improved with increasing average number of records per species for two of the three SDM methods, and the improvement in Kappa decelerated as the number of records per species increased (Fig. S5).

\subsubsection{Effect of spatial bias}

Higher levels of spatial sampling bias generally reduced AUC and Kappa, but the size of this effect was small for the low level of bias (Fig. 6). SDMs built with GLMs showed the biggest difference in prediction performance between models trained with unbiased data and models trained with data showing median spatial bias (reduction in expected AUC of 0.037 when using an average of 200 records per species, Fig. 6). Other SDM methods showed less difference in AUC between models trained with unbiased data and models trained with data containing median spatial bias (decrease in expected AUC of 0.033 for boosted regression trees and 0.030 for inverse distance-weighted interpolation when using an average of 200 records per species). The AUC for inverse distance-weighted interpolation models trained with unbiased data was generally higher than the AUC for GLMs and boosted regression trees trained with severely biased data, but lower than the AUC for GLMs and boosted regression trees trained with data with median spatial bias for any given sample size (Fig. 6, Fig. 7). 


\section{DISCUSSION}

358 Both sample size (the average number of observations per species) and choice of modelling

359 method were more important than the spatial bias of training data for determining model

360 prediction performance. This is in line with the results of Thibaud et al. (2014), and the

361 importance of sample size was in line with studies summarized in Meynard, Leroy \& Kaplan

362 (2019). Thibaud et al. (2014) simulated spatial sampling bias by defining sampling probability

363 as a linear function of distance from the nearest road. In contrast, our study used observed

364 spatial sampling patterns from real biological records datasets. Our results therefore provide a

365 more direct confirmation that spatial biases of the type and intensity found in real datasets are not

366 as important as other factors in determining SDM prediction performance. We did not measure

367 how different our biased sampling was from Thibaud et al.'s (2014) biased sampling, but we

368 suspect biases in real data are more severe than the bias tested by Thibaud et al. (2014). More

369 attempts to quantify sampling biases in real data would be valuable for informing how bias is

370 incorporated into future simulation studies.

371 While spatial bias was not the most important factor determining SDM prediction performance,

372 spatial sampling bias did affect model prediction performance when spatial bias was relatively

373 strong. The limited effect of spatial bias on SDMs that we observed is similar to other findings

374 that have shown spatial sampling bias to have a small effect on model performance (Thibaud et

375 al., 2014; Warton et al., 2013) or to affect only some SDM methods (Barbet-Massin et al., 2012).

376 Our study used spatial biases and the spatially explicit environmental data representative of data

377 likely to be used in SDMs using biological records in Ireland. Our conclusions therefore apply

378 most directly to applications of SDMs using Irish biological records, and may not be

379 generalizable to other geographic locations, or for species within Ireland that do not respond to 
380 the environmental predictor variables used in this study. Our simulations could be "scaled up" to

381 use environmental variables from, and create virtual species over, a larger spatial extent, which

382 would provide insight about whether spatial sampling bias remains relatively un-important at

383 larger (e.g. continental) scales. However, our results strengthen a growing body of literature that

384 suggests that spatial sampling bias is rarely the most important issue in determining SDM

385 prediction performance. In particular, the choice of modelling method may often have more

386 impact on SDM prediction performance than a variety of other factors (Barbet-Massin et al.,

387 2012; Fernandes, Scherrer, \& Guisan, 2018).

388 Training data with low spatial sampling bias produced species distribution models that

389 performed nearly as well as models trained with unbiased data, when the task was ranking the

390 probability of a species being detected at sites. Prediction performance was poor when models

391 were trained with small sample sizes, regardless of the spatial bias in training data. Similarly,

392 model performance increased quickly with sample size when sample size was small, even when

393 the data had severe spatial bias. This suggests that, for taxonomic groups with relatively few

394 records per species, the usefulness of the data for predictive SDMs can be improved by

395 increasing sample size, even if additional data collection is spatially biased. In contrast, for

396 taxonomic groups for which biological records datasets already have a high average number of

397 records per species (e.g. birds and butterflies which both have an average of over 2000 records

398 per species in Ireland) further improvements in SDM prediction performance will likely require

399 increasing the spatial evenness of data (Fig. 9).

400 While our SDMs were able to correctly rank sites in terms of the probability of a focal species

401 being detected (measured using AUC), the SDMs were essentially unable to convert the

402 continuous SDM outputs into binary presence/absence maps (measured using Cohen’s Kappa) 
403 for locations outside the training data (Fig. S6). Converting continuous SDM outputs to binary

404 classifications is fraught with difficulties, and the criteria that should be used for selecting the

405 threshold for conversion depend on the purpose of the SDMs (Guillera-Arroita et al., 2015, and

406 references therein). Any conversion of a continuous SDM output to a binary classification

407 necessarily results in a loss of information, and Guillera-Arroita et al. (2015) suggest that for

408 most applications of SDMs, binary conversions are not necessary. It was outside the scope of

409 this study to explore why SDMs were better at ranking than at producing binary classifications

410 for sites outside the training data. The main finding of our study, that spatial sampling bias was

411 less important than other factors in determining model performance, was consistent regardless of

412 whether prediction performance was evaluated in terms of the ability to rank (AUC) or classify

413 (Kappa) sites (Table 3).

414 Presence-only data are common in biological records, but there are very few true "presence-

415 only" SDM modeling methods. Most common SDM methods require information on presences

416 and something else (e.g. non-detections, or background-, quadrature-, or pseudo-absences

417 points) (Phillipes et al., 2009; Elith, Phillips, Hastie, Dudik, Chee \& Yates, 2011; Renner et al.,

418 2015). We inferred non-detections from detections of other species (e.g. Phillips et al., 2009;

419 van Strien, Termaat, Groenendijk, Mensing, \& Kéry, 2010; Isaac, van Strien, August, de Zeeuw,

420 \& Roy, 2014; Johnston et al. 2020). Other options include generating pseudo-absences randomly

421 or according to a variety of rules (Barbet-Massin et al. 2012). Phillips et al. (2009) proposed a

422 "target group" approach that selects background points using detections of other species. The

423 target group approach is essentially identical to ours. Our results - particularly the finding that

424 spatial sampling bias was relatively un-important relative to other factors - applies most directly

425 to SDM approaches that infer non-detections from presence records of other species. When 
426 background or pseudo-absence points are generated randomly or using an ad-hoc rule, we expect

427 that SDMs will be more strongly impacted by spatial bias in the presence-only data than were

428 SDMs in our study (Phillips et al. 2009).

429 The objective of our SDMs was to fill in gaps in species distribution knowledge within the 430 spatial and environmental conditions of the island of Ireland, an area of about $84,000 \mathrm{~km}^{2}$. Our

431 results may not generalize to larger spatial scales or to cases in which the goal of SDMs is

432 uncovering species' entire fundamental environmental niche or determining the environmental

433 factors most strongly influencing distributions. The spatial scope of our SDMs is sensible both

434 from an ecological and applied standpoint, because the island of Ireland is a geographically

435 delimited ecological unit, and because decision making about species conservation and

436 management often happens within political units (e.g. nations, states, or counties) that cover only

437 a portion of species' spatial and environmental distributions.

438 GLMs had the best prediction performance of the SDM methods we tested, even though they 439 were more affected by spatial bias than were other methods. The high performance of GLMs

440 relative to other modelling methods in this study agrees with the simulation results of Thibaud et 441 al. (2014) and Fernandes et al. (2018). However, as in both those studies, we generated virtual

442 species distributions according to a linear model, so it is possible that the good performance of

443 GLMs is due to the model having the same functional form as the "true" species responses. In 444 real applications, it is unlikely that the functional form of the model will exactly match the form

445 of the true species responses. Indeed, the species distribution modelling literature has many 446 examples of different modelling methods performing best in different studies, suggesting that no

447 modelling method consistently outperforms others (Bahn \& McGill, 2007; Breiner, Nobis,

448 Bergamini, \& Guisan, 2018; Cutler et al., 2007; Elith et al., 2006; Elith \& Graham 2009). 
450 GLMs', and prediction performance of both methods was similar when trained with large,

451 spatially biased datasets. But boosted regression trees failed to fit models more often than did

452 GLMs, especially when sample sizes were smaller, which may make them inferior to other

453 modelling methods for small datasets, at least within the computational resource limits we faced.

454 We cannot rule out the possibility that the performance of boosted regression trees would

455 improve if they were trained with a smaller learning rate and permitted to grow more than 30,000

456 trees. However, most users of SDMs will face some computational resource limitations.

457 In this study, we introduced spatial bias specifically into the training data and tested model

458 performance using spatially even evaluation data. However, spatial bias can also occur in

459 evaluation data and may affect the reliability of model evaluations (Fink et al., 2010). When

460 using real biological records datasets, it is likely that both model training and evaluation will use

461 spatially biased data, making it difficult to dis-entangle whether observed effects of spatially

462 biased data on prediction performance are due to the influence of biased data in the model

463 training step or in the model evaluation step. We evaluated models on spatially even data (which

464 is easy using simulated data but would be more difficult or impossible when using real data), so

465 the observed effects of spatially biased data on prediction performance in our study can be

466 attributed to the effect of biased data on model training. All of the SDM methods we used

467 involve some kind of model evaluation as part of the model training process, either inherent in

468 the model fitting or introduced by our implementation. For example, with our GLMs we

469 introduced a model evaluation step to select predictor variables. The final GLM models were

470 therefore based on variables that had been selected by evaluation on spatially biased data. For

471 both GLMs and inverse distance-weighted interpolation, it is possible that using unbiased data in 
472 the evaluations during model selection would have led to different final models. Therefore, the

473 observed effect of the spatial bias in this study could be due to how biased data affects the actual

474 fitting of each individual model, or to how the biased data affects the evaluation step used to

475 select which fitted model to use for predictions. Tree-based methods, including boosted

476 regression trees, select which values of predictor variables to split at and/or which predictor

477 variables to use at each node based on how much those splits improve some measure of

478 performance on the training data (Elith et al., 2008; Hastie et al., 2009). Thus, evaluation on

479 potentially spatially biased training data is inherent in fitting tree models.

480 Fink et al. (2010) provided a method for correcting spatial bias in evaluation data to reduce the

481 effect of spatial bias on model evaluation, but they did not explicitly address spatially biased data

482 in model training. Our results showed that spatially biased data can impact model training (at

483 least when the spatial bias is relatively strong). Investigating the effect of spatially biased data on

484 the evaluation that takes place as part of model training (e.g. during variable selection or

485 parameter tuning) may be a worthwhile path for future research. It may be possible to use a

486 method like that proposed by Fink et al. (2010) to correct spatial bias during the evaluation that

487 takes place within the model training process.

\section{CONCLUSION}

490 We found that spatial sampling bias in training data affected species distribution model

491 prediction performance when the spatial bias was relatively strong, but that sample size and the

492 choice of modelling method were more important than spatial bias in determining model

493 prediction performance. This study adds to a body of literature suggesting that prediction 
494 performance of species distribution models is less affected by spatial sampling bias in training

495 data than by other factors including modelling method and sample size. We suggest that, when

496 biological records datasets are relatively small, model prediction performance can best be

497 improved by increasing the number of records, even if additional data are sampled with spatial

498 bias. Attempts to reduce spatial bias in data through data filtering should be cautious about the

499 resulting decrease in sample size, which could cancel any gains in prediction performance from

500 reducing spatial sampling bias. Converting continuous SDM outputs to binary maps should be

501 done with caution, and prediction performance tests should use spatial block cross-validation or

502 test on independent data.

\section{$503 \quad 6$ ACKNOWLEDGMENTS}

504 We thank Tomás Murray and the Irish National Biodiversity Data Centre (NBDC) for

505 providing and answering questions about the biological records data, and we thank the many

506 citizen scientists who collected and contributed their data to the NBDC. This work used the

507 ResearchIT Sonic cluster funded by UCD IT Services and the Research Office. This research

508 used CORINE data made available with funding by the European Union. 


\section{REFERENCES}

513 Amano, T., \& Sutherland, W. J. (2013). Four barriers to the global understanding of biodiversity

514 conservation: Wealth, language, geographical location and security. Proceedings of the Royal

$515 \quad$ Society B: Biological Sciences, 280, 20122649.

516 Amano, T., Lamming, J. D. L., \& Sutherland, W. J. (2016). Spatial gaps in global biodiversity

517 information and the role of citizen science. BioScience, 66, 393-400.

518 Amante, C., \& Eakins, B. W. (2009). ETOPO1 1 arc-minute global relief model: Procedures,

519 data sources and analysis. NOAA Technical Memorandum NESDIS NGDC-24. National

520 Geophysical Data Center, NOAA. doi: 10.7289/V5C8276M [accessed 8 May 2019].

521

522 Bahn, V., \& McGill, B. J. (2007). Can niche-based distribution models outperform spatial 523 interpolation? Global Ecology and Biogeography, 16, 733-742.

524 Barbet-Massin, M., Jiguet, F., Albert, C. H., \& Thuiller, W. (2012). Selecting pseudo-absences 525 for species distribution models: How, where and how many? Methods in Ecology and 526 Evolution, 3, 327-338.

527 Bivand, R., Keitt, T., \& Rowlingson, B. (2018). rgdal: Bindings for the 'geospatial' data 528 abstraction library. $\mathrm{R}$ package versions 1.3-9 and 1.4-4.

529 Boakes, E. H., McGowan, P. J. K., Fuller, R. A., Chang-qing, D., Clark, N. E., O’Connor, K., \& 530 Mace, G. M. (2010). Distorted views of biodiversity: Spatial and temporal bias in species 531 occurrence data. PLoS Biology, 8, e1000385.

532 Breiner, F. T., Nobis, M. P., Bergamini, A., \& Guisan, A. (2018). Optimizing ensembles of small 533 models for predicting the distribution of species with few occurrences. Methods in Ecology 534 and Evolution, 9, 802-808. 
535 Carvell, C., Isaac, N. J. B., Jitlal, M., Peyton, J., Powney, G. D., Roy, D. B., ... Roy, H. E.

536 (2016). Design and testing of a national pollinator and pollination monitoring framework.

537 Final summary report to the Department for Environment, Food and Rural Affairs (Defra),

538 Scottish Government; Welsh Government: Project WC1101.

539 Cohen, J. (1960). A coefficient of agreement for nominal scales. Educational and Psychological

$540 \quad$ Measurement, 20, 37-46.

541 CORINE land cover database. (2012). Version 18. (C) European Union, Copernicus Land

542 Monitoring Service 2016, European Environment Agency (EEA). Retreived from

543 https://www.eea.europa.eu/ds_resolveuid/ecb838dabf4849838ba5f3dc81ca6b0e [8 Aug 2016].

544 Cressie, N. A. C. (1991). Statistics for spatial data. New York: John Wiley \& Sons, Inc.

545 Cutler, D. R., Edwards, T. C., Beard, K. H., Cutler, A., Hess, K. T., Gibson, J., \& Lawler, J. J.

546 (2007). Random forests for classification in ecology. Ecology, 88, 2783-2792.

547 Deka, M., \& Morshed, N. (2018). Mapping disease transmission risk of Nipah Virus in South

548 and Southeast Asia. Tropical Medicine and Infectious Disease, 3, 57.

549 Dray, S., \& Dufour, A. (2007). The ade4 package: Implementing the duality diagram for

550 ecologists. Journal of Statistical Software, 22, 1-20.

551 Dyer, R. J., Gillings, S., Pywell, R. F., Fox, R., Roy, D. B., \& Oliver, T. H. (2017). Developing a

552 biodiversity-based indicator for large-scale environmental assessment: A case study of

553 proposed shale gas extraction sites in Britain. Journal of Applied Ecology, 54, 872-882.

554 Edwards, T. C., Cutler, D. R., Zimmermann, N. E., Geiser, L., \& Moisen, G. G. (2006). Effects

555 of sample survey design on the accuracy of classification tree models in species distribution 556 models. Ecological Modelling, 199, 132-141. 
557 Elith, J., \& Graham, C. H. (2009). Do they? How do they? Why do they differ? On finding

558 reasons for differing performances of species distribution models. Ecography, 32, 66-77.

559 Elith, J., Graham, C. H., Anderson, R. P., Dudík, M., Ferrier, S., Guisan, A., ... Zimmermann, N.

560 E. (2006). Novel methods improve prediction of species' distributions from occurrence data.

561 Ecography, 29, 129-151.

562 Elith, J., Leathwick, J. R., \& Hastie, T. (2008). A working guide to boosted regression trees. 563 Journal of Animal Ecology, 77, 802-813.

564 Elith, J., Phillips, S. J., Hastie, T., Dudik, M., Chee, Y. E., Yates, C. J. (2011). A statistical 565 explanation of MaxEnt for ecologists. Diversity and Distributions, 17, 43-57.

566 Fan, W., Davidson, I., Zadrozny, B., \& Yu, P. S. (2005). An improved categorization of

567 classifier's sensitivity on sample selection bias. In Fifth IEEE International Conference on

568 Data Mining (ICDM'05), Houston, TX: IEEE.

569 Fernandes, R. F., Scherrer, D., \& Guisan, A. (2018). How much should one sample to accurately

570 predict the distribution of species assemblages? A virtual community approach. Ecological

$571 \quad$ Informatics, 48, 125-134.

572 Fink, D., Hochachka, W. M., Zuckerberg, B., Winkler, D. W., Shaby, B., Munson, M. A., ...

573 Kelling, S. (2010). Spatiotemporal exploratory models for broad-scale survey data. Ecological 574 Applications, 20, 2131-2147.

575 Friedman, J. H. (2001). Greedy function approximation: A gradient boosting machine. The 576 Annals of Statistics, 29, 1189-1232.

577 Gelman, A., \& Hill, J. (2006). Data analysis using regression and multilevel/hierarchical models 578 (Analytical Methods for Social Research). Cambridge: Cambridge University Press. 
579 Gräler, B., Pebesma, E., \& Heuvelink, G. (2016). Spatio-temporal interpolation using gstat. The $580 \quad$ R Journal, 8, 204-218.

581 Greenwell, B., Boehmke, B., Cunningham, J., \& GBM Developers. (2018). gbm: Generalized $582 \quad$ boosted regression models. $\mathrm{R}$ package version 2.1.4.

583 Guillera-Arriota, G., Lahoz-Monfort, J. J. Elith, J. Gordon, A. Kujala, H., Lentini, P. E.,

584 McCarthy, M. A., Tingley, R., Wintle, B. A. (2015). Is my species distribution model fit for 585 purpose? Matching data and models to applications. Global Ecology and Biogeography, 24, $586 \quad 276-292$.

587 Hastie, T., Tibshirani, R., \& Friedman, J. (2009). The elements of statistical learning: Data 588 mining, inference and prediction (2nd ed.). New York: Springer.

589 Haylock, M. R., Hofstra, N., Klein Tank, A. M. G., Klok, E. J., Jones, P. D., \& New, M. (2008). 590 A European daily high-resolution gridded data set of surface temperature and precipitation for 591 1950-2006. Journal of Geophysical Research, 113, D20119.

592 Hijmans, R. J. (2018). raster: Geographic data analysis and modeling. R package versions 2.8-4 593 and 2.9-23.

594 Hijmans, R. J., Phillips, S., Leathwick, J., \& Elith, J. (2017). dismo: Species distribution 595 modeling. R package version 1.1-4.

596 Hosmer, D. W., \& Lemeshow, S. (2000). Applied logistic regression (2nd ed). New York: 597 Wiley.

598 Isaac, N. J. B., and Pocock, M. J. O. (2015). Bias and information in biological records. 599 Biological Journal of the Linnean Society, 115, 522-531. 
600 Isaac, N. J. B., van Strien, A. J., August, T. A., de Zeeuw, M. P., \& Roy, D. B. (2014). Statistics

601 for citizen science: Extracting signals of change from noisy ecological data. Methods in

602 Ecology and Evolution, 5, 1052-1060.

603 Johnston, A., Moran, N., Musgrove, A., Fink, D., \& Baillie, S. R. (2020). Estimating species

604 distributions from spatially biased citizen science data. Ecological Modelling, 422, 108927.

605 https://doi.org/10.1016/j.ecolmodel.2019.108927

606 Kéry, M., Royle, A., Schmid, H., Schaub, M., Volet, B., Häfliger, G., \& Zbinden, N. (2010).

607 Site-occupancy distribution modeling to correct population-trend estimates derived from

608 opportunistic observations. Conservation Biology, 24, 1388-1397.

609 Leroy, B., Meynard, C. N., Bellard, C., \& Courchamp, F. (2016). virtualspecies, an R package to 610 generate virtual species distributions. Ecography, 39, 599-607.

611

612 Magurran, A. E., \& McGill, B. J. (Eds.). (2011). Biological diversity: Frontiers in measurement 613 and assessment. Oxford: Oxford University Press.

614 Meynard, C. N., Leroy, B., \& Kaplan, D. M. (2019). Testing methods in species distribution

615 modelling using virtual species: what have we learnt and what are we missing?. Ecography,

$616 \quad 42,2021-2036$.

617 Nakagawa, S. (2015). Missing data: mechanisms, methods, and messages. In G. A. Fox, S.

618 Negrette-Yankelevich, \& V. J. Sosa (Eds.), Ecological statistics: Contemporary theory and

619 application (First Ed., pp. 81-105). Oxford: Oxford University Press.

620 Newson, S. E., Evans, H. E., Gillings, S., Jarrett, D., Raynor, R., \& Wilson, M. W. (2017).

621 Large-scale citizen science improves assessment of risk posed by wind farms to bats in

622 southern Scotland. Biological Conservation, 215, 61-71. 
623 Oliveira, U., Paglia, A. P., Brescovit, A. D., de Carvalho, C. J. B., Silva, D. P., Rezende, D. T., ...

624 Santos, A. J. (2016). The strong influence of collection bias on biodiversity knowledge

625 shortfalls of Brazilian terrestrial biodiversity. Diversity and Distributions, 22, 1232-1244.

626 Pebesma, E. (2018). Simple features for R: Standardized support for spatial vector data. The R

627 Journal, 10, 439-446.

628 Pebesma, E. J. (2004). Multivariable geostatistics in S: The gstat package. Computers \& 629 Geosciences, 30, 683-691.

630 Phillips, S. J., Dudík, M., Elith, J., Graham, C. H., Lehmann, A., Leathwick, J., \& Ferrier, S.

631 (2009). Sample selection bias and presence-only distribution models: Implications for

632 background and pseudo-absence data. Ecological Applications, 19, 181-197.

633 R Core Team (2020). R: A language and environment for statistical computing. Vienna, Austria:

634 R Foundation for Statistical Computing.

635 Redding, D. W., Atkinson, P. M., Cunningham, A. A., Lo Iacono, G., Moses, L. M., Wood, J. L.

636 N., \& Jones, K. E. (2019). Impacts of environmental and socio-economic factors on

637 emergence and epidemic potential of Ebola in Africa. Nature Communications, 10, 4531.

638 Renner, I. W., Elith, J., Baddeley, A., Fithian, W. Hastie, T., Phillips, S. J., Popovic, G., \&

639 Warton, D. I. (2015). Point process models for presence-only analysis. Methods in Ecology

$640 \quad$ and Evolution, 6, 366-379.

641 Roberts, D. R., Bahn, V., Ciuti, S., Boyce, M. S., Elith, J., Guillera-Arroita, G., ... Dormann, C.

642 F. (2017). Cross-validation strategies for data with temporal, spatial, hierarchical, or

643 phylogenetic structure. Ecography, 40, 913-929.

644 Ross, N. (2018). fasterize: Fast polygon to raster conversion. R package version 1.0.0. 
645 Simmonds, E. G., Jarvis, S. G., Henrys, P. A., Isaac, N. J. B., \& O’Hara, R. B. (2020). Is more

646 data always better? A simulation study of benefits and limitations of integrated distribution

647 models. Ecography, 43, 1-10.

648 Stolar, J., \& Nielsen, S. E. (2015). Accounting for spatially biased sampling effort in presence-

649 only species distribution modelling. Diversity and Distributions, 21, 595-608.

650 Thibaud, E., Petitpierre, B., Broennimann, O., Davison, A. C., \& Guisan, A. (2014). Measuring

651 the relative effect of factors affecting species distribution model predictions. Methods in

652 Ecology and Evolution, 5, 947-955.

653 Uzarski, D. G., Brady, V. J., Cooper, M. J., Wilcox, D. A., Albert, D. A., Axler, R. P., ...

654 Schneider, J. P. (2017). Standardized measures of coastal wetland condition: Implementation

655 at a Laurentian Great Lakes basin-wide scale. Wetlands, 37, $15-32$.

656 van den Besselaar, E. J. M., Haylock, M. R., van der Schrier, G., \& Klein Tank, A. M. G. (2011).

657 A European daily high-resolution observational gridded data set of sea level pressure. Journal

658 of Geophysical Research Atmospheres, 116, D11110.

659 van Strien, A. J., van Swaay, C. A. M. \& Termaat, T. (2013). Opportunistic citizen science data

660 of animal species produce reliable estimates of distribution trends if analysed with occupancy

661 models. Journal of Applied Ecology, 50, 1450-1458.

662 van Strien, A. J., Termaat, T., Groenendijk, D., Mensing, V., \& Kéry, M. (2010). Site-occupancy

663 models may offer new opportunities for dragonfly monitoring based on daily species lists.

664 Basic and Applied Ecology, 11, 495-503.

665 Warton, D. I., Renner, I. W., \& Ramp, D. (2013). Model-based control of observer bias for the

666 analysis of presence-only data in ecology. PLOS ONE, 8, e79168.

667 Wickham, H. (2017). tidyverse: Easily install and load the 'tidyverse'. R package version 1.2.1. 
668 Wooldridge, J. M. (2009). Introductory econometrics: A modern approach (4th ed.). Mason, OH:

669 South-Western.

670 Zurell, D., Berger, U., Cabral, J. S., Jeltsch, F., Meynard, C. N., Münkemüller, T., ... Grimm, V. 671 (2010). The virtual ecologist approach: Simulating data and observers. Oikos, 119, 622-635. 


\section{Figure 1}

Simulation and analysis process.

Step 1 - define spatial sampling biases: we used the locations of biological records of three different taxa in Ireland to produce rasters of the observed probability of sampling from each $10 \times 10 \mathrm{~km}$ grid cell in Ireland. Step 2 - generate virtual species true distributions: the probability of virtual species occurring in each grid cell was defined as a response to real environmental variables. True presence/absence distributions were generated probabilistically. Step 3 - sample virtual species with spatial biases: detection-only observations of species were drawn from sampling locations selected according to the spatial sampling bias rasters generated in Step 1, to create a dataset of virtual detection-only biological records. Step 4 - Infer non-detections using detections of other species: nondetections of each species were inferred at locations where other species had been recorded, transforming the detection-only virtual biological records into detection/non-detection data. Step 5 - train SDMs: SDMs were trained with the virtual detection/non-detection biological records as the response variable and the real environmental variables as predictors. Step 6 test SDMs: SDM prediction performance was assessed using three metrics, which capture different aspects of models prediction performance. Steps 5 and 6 (blue bounding box) were conducted using a spatial block cross-validation framework illustrated in Fig. 3. 
Step 1

Define spatial sampling biases

\author{
Real biological \\ records data
}

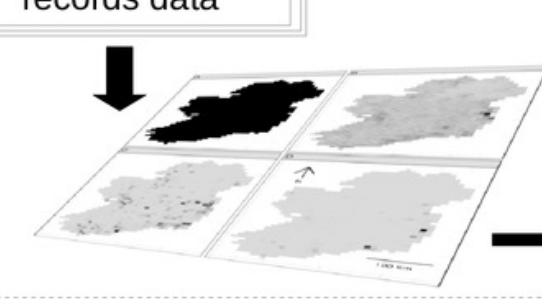

Four sampling bias rasters (Fig. 2) defining the probability of sampling from each grid cell
Step 2 Generate virtual species true distributions
Step 3

Sample virtual species with spatial biases
Real environmental

data

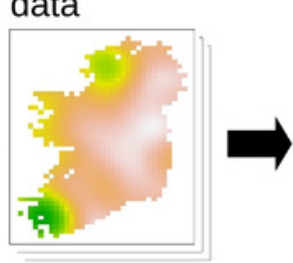

Generate virtual species distributions according to a linear model. The probability of the species occurring is a function of environmental predictors:

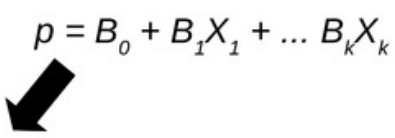

True virtual species distributions (Fig. 4)
Step 4

Infer non-detections using detections of other species

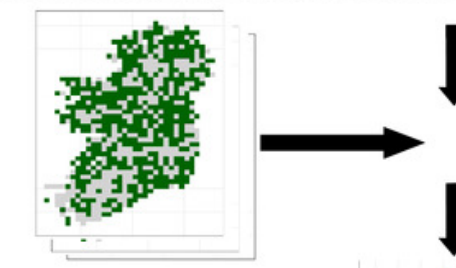

Sample presence-only records of virtual species from locations defined by the sampling bias rasters

Presence-only virtual biological records

Detection/non-detection virtual biological records

\section{Step 5} Train SDMs

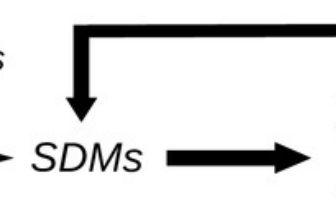

Continuous predictions of relative probability of detecting the species

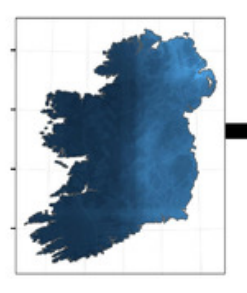

Step 6

Test SDMs

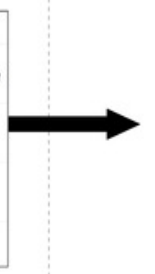

Tests how well predicted distributions match true distributions

AUC Max Kappa RMSE 


\section{Figure 2}

Spatial sampling patterns from Irish biological records.

Spatial sampling patterns from Irish biological records were used as templates to create virtual species records data with varying amounts of spatial bias. Darker shades indicate higher relative probability of sampling from a grid square compared to other grid squares within the same template; overall sampling effort is the same for each panel (A) through (D). The most heavily sampled grid square in each spatial bias template has a relative recording effort of one, while a grid square with half as many records as the most heavily sampled square has a relative recording effort of 0.5 . Spatial sampling patterns derived from datasets for different taxonomic groups were: (A) no bias (even probability of sampling from every grid square), (B) low bias (based on bird data), (C) median bias (based on butterflies), and (D) severe bias (based on moths). 

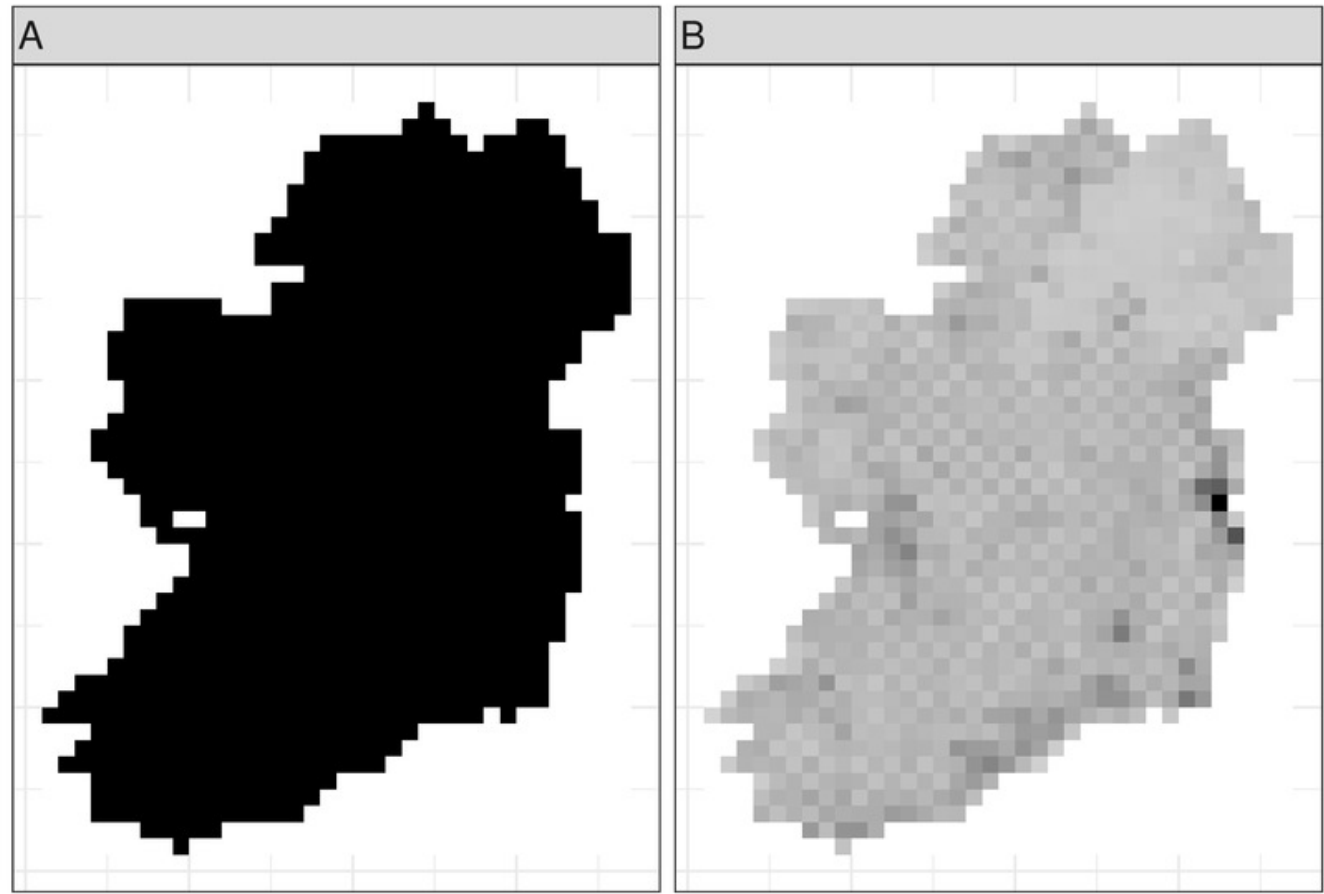

Relative

Recording
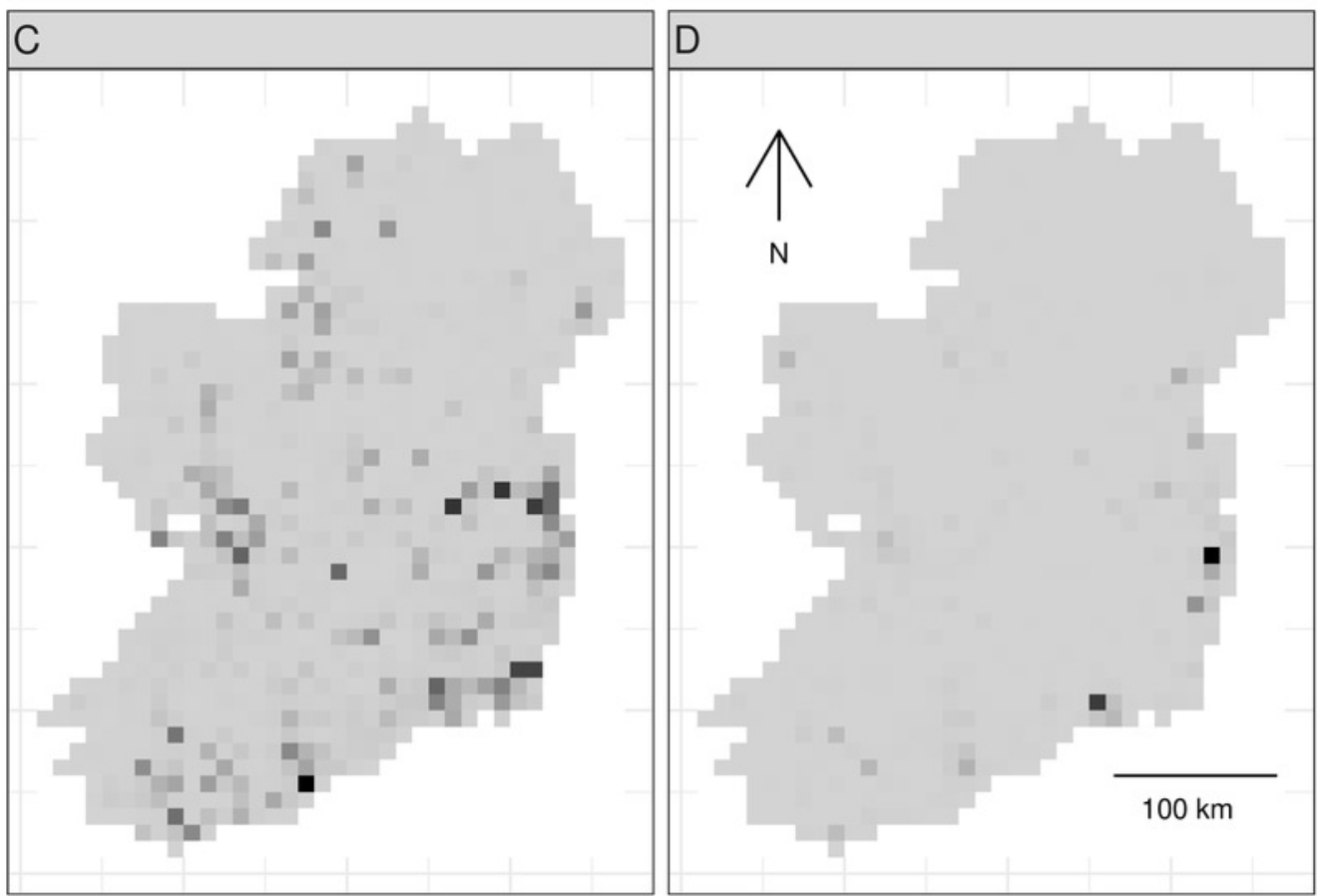

Effort

1.00

0.75

0.50

0.25

0.00 


\section{Figure 3}

Species distribution model training and testing process for a single cross-validation fold.

The true virtual species distribution (A, presences shown in dark green, absences in light grey) was sampled to produce virtual biological records with a range of sample sizes and spatial biases, including no bias (B) and median bias (C). Orange points in (B) and (C) show checklists on which the species was recorded, black points show checklists on which the species was not recorded (i.e. non-detection points). Species distribution models were fit using five-fold spatial block cross validation, in which data from about $80 \%$ of the spatial area was used to train models (light grey background in B and C). Data from the remaining spatial areas (dark grey background in B and C) was set aside for model evaluation. Model evaluation tested the ability of species distribution models to predict the true presence (orange dots) or absence (black dots) of the species in each grid cell within the evaluation areas (D). Model evaluation therefore used spatially even data with the same number of evaluation points (D) regardless of the sample size and spatial bias of training data (B and C). 


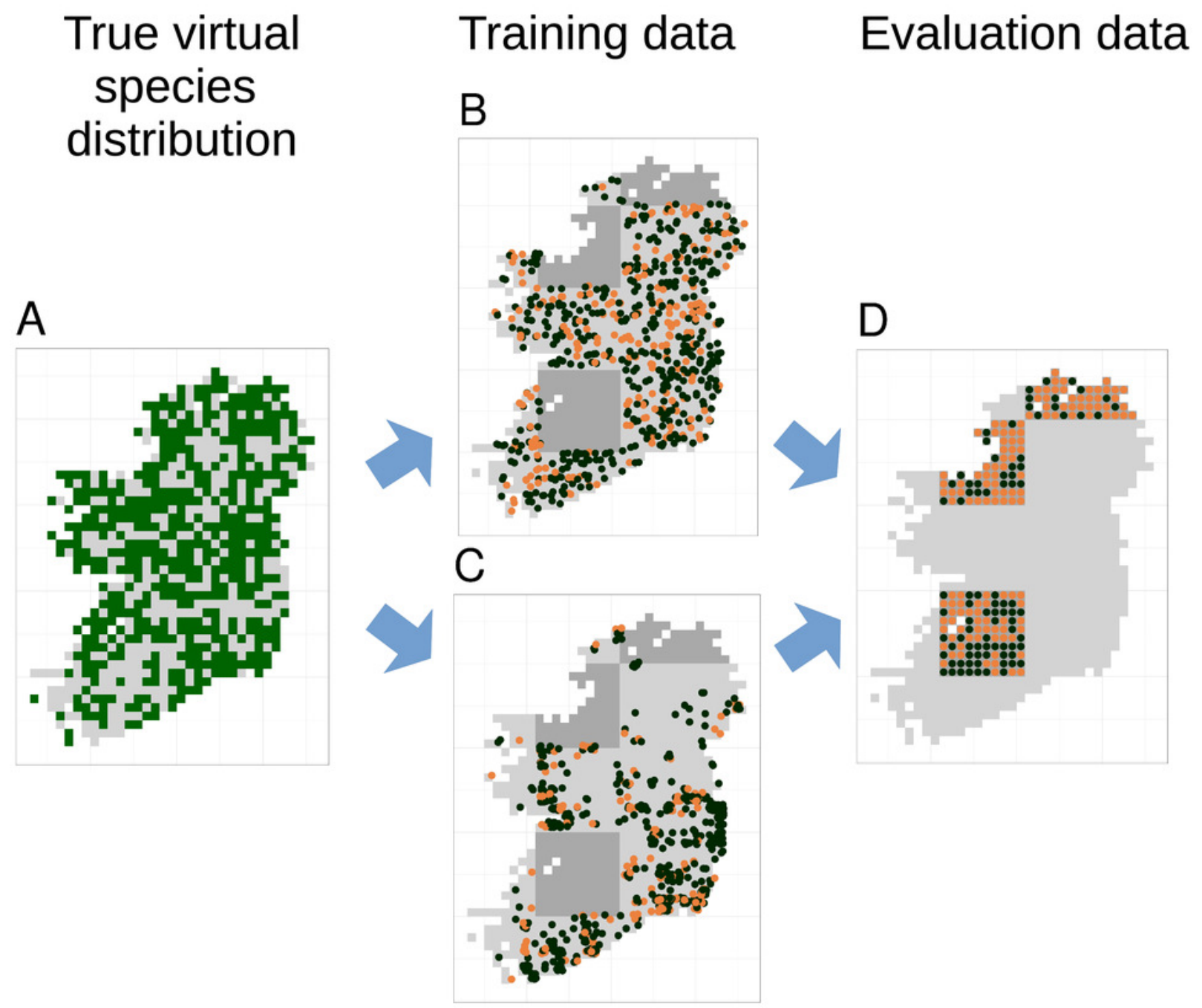




\section{Figure 4}

The true distributions of four example simulated species.

Simulated species showed a range of plausible distributions with a range of prevalences, including (A) common widespread species, (B) rare species mostly limited to north-western coastal sites, (C) species with a north/south gradient in occurrence, and (D) common species that are absent from southern sites. 
A

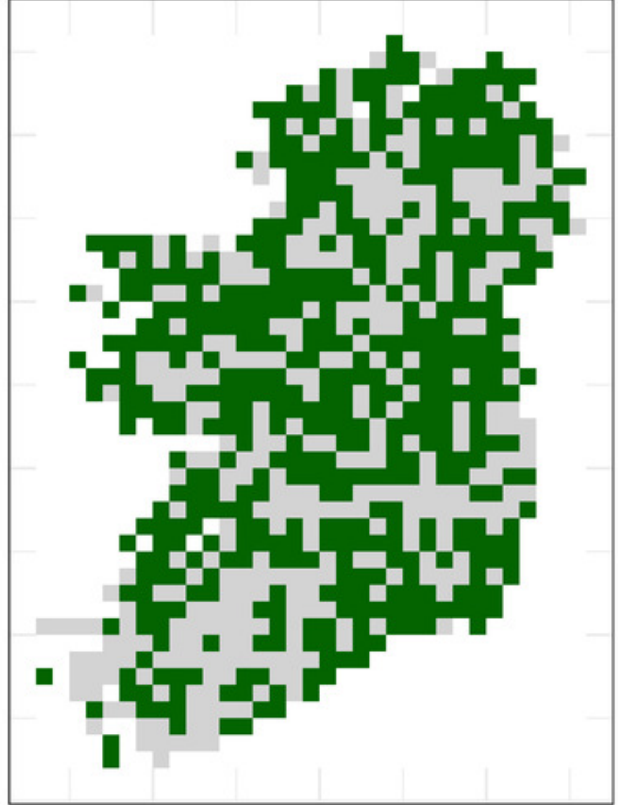

C

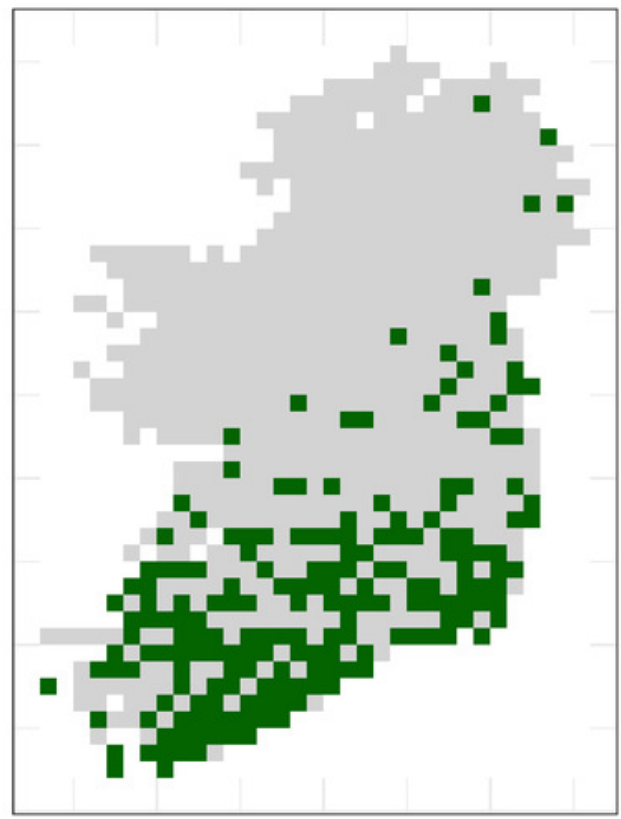

B

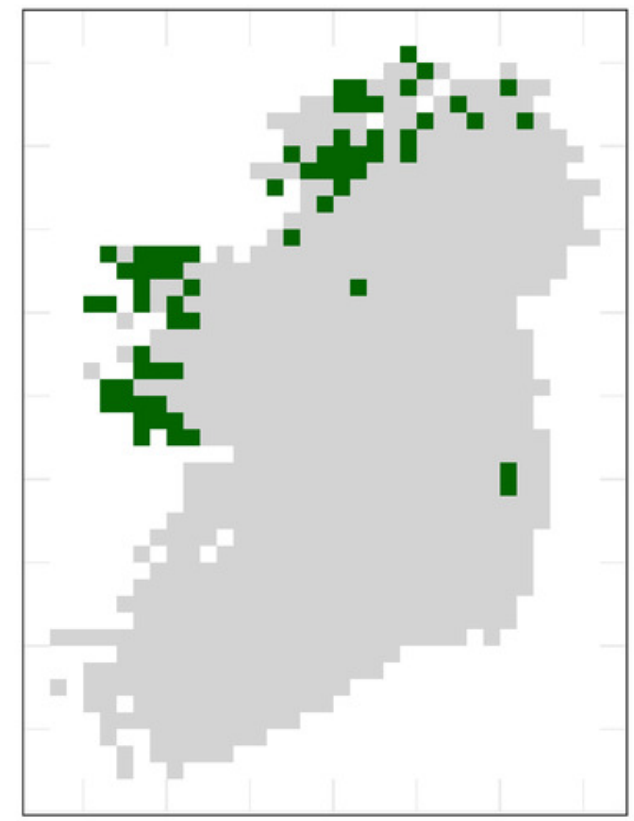

D

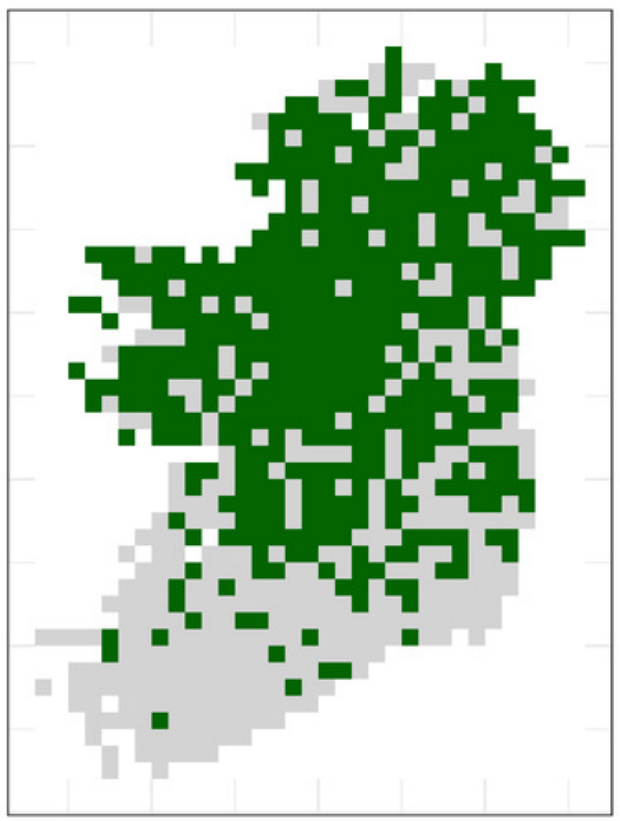




\section{Figure 5}

The number of virtual species successfully modeled.

The number of virtual species (out of 110 total species chosen for modelling from the large community simulation) for which species distribution models fitted within the computation time and memory constraints we imposed, according to the spatial sampling bias and sample size of training data and the species distribution modelling method. Species distribution modelling methods were (A) generalized linear models, (B) boosted regression trees, and (C) inverse distance-weighted interpolation. Spatial biases were no bias (Simpson evenness $=1$ ), low (e.g. birds, Simpson evenness $=0.76$ ), median (e.g. butterflies, Simpson evenness $=$ 0.13 ), and severe (e.g. moths, Simpson evenness $=0.02$ ). 


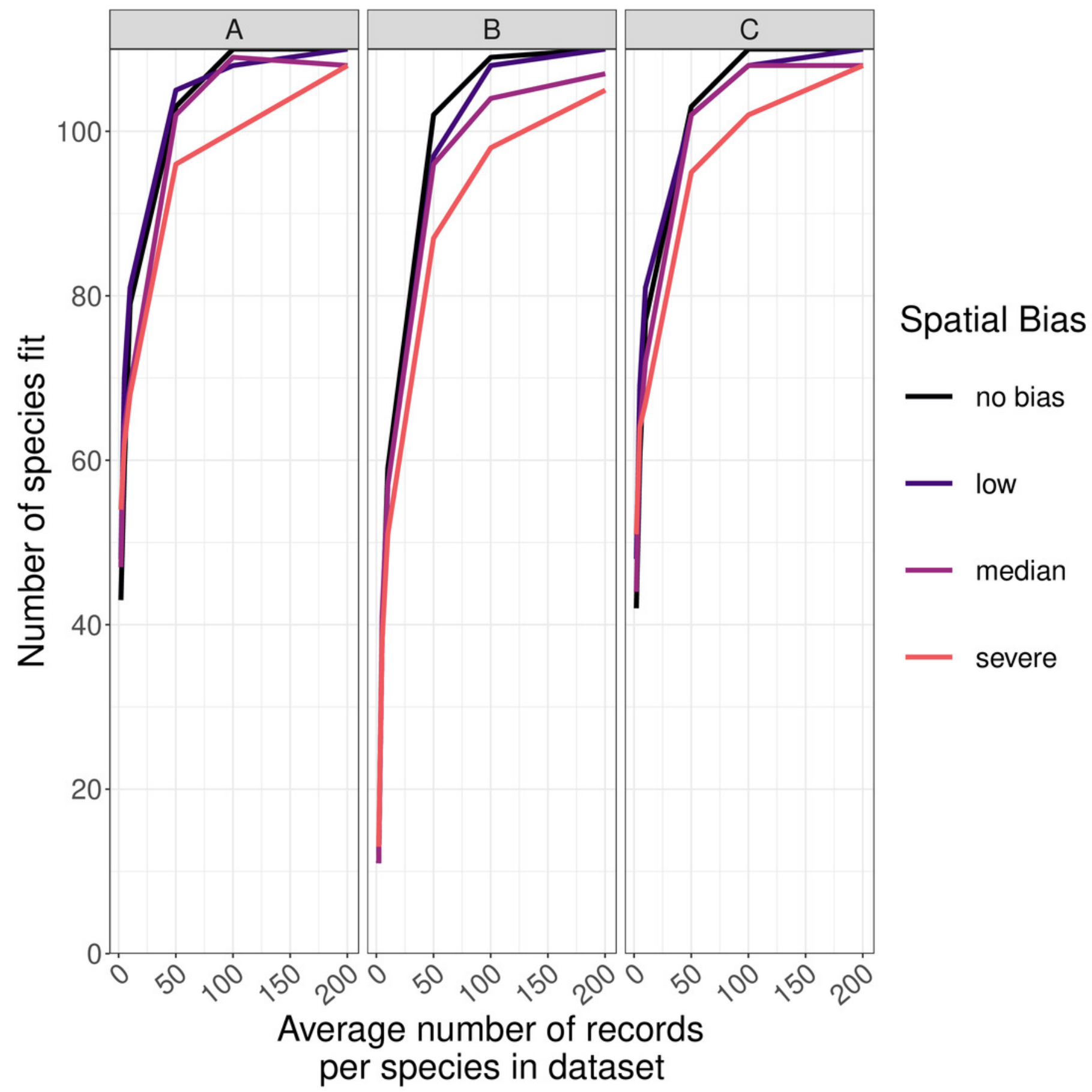




\section{Figure 6}

Expected prediction performance of species distribution models for 110 simulated species under a range of sample size and spatial sampling bias scenarios.

Panels show the expected prediction performance of species distribution models constructed using (A) generalize linear models, (B) boosted regression trees, and (C) inverse distanceweighted interpolation. Lines show expected area under the receiver operating characteristic curve (AUC) given the sample size and spatial sampling bias of training data, and the species distribution modelling method. Rug plots indicate sample sizes (mean number of records per species) of the virtual biological records datasets used to train species distribution models. 


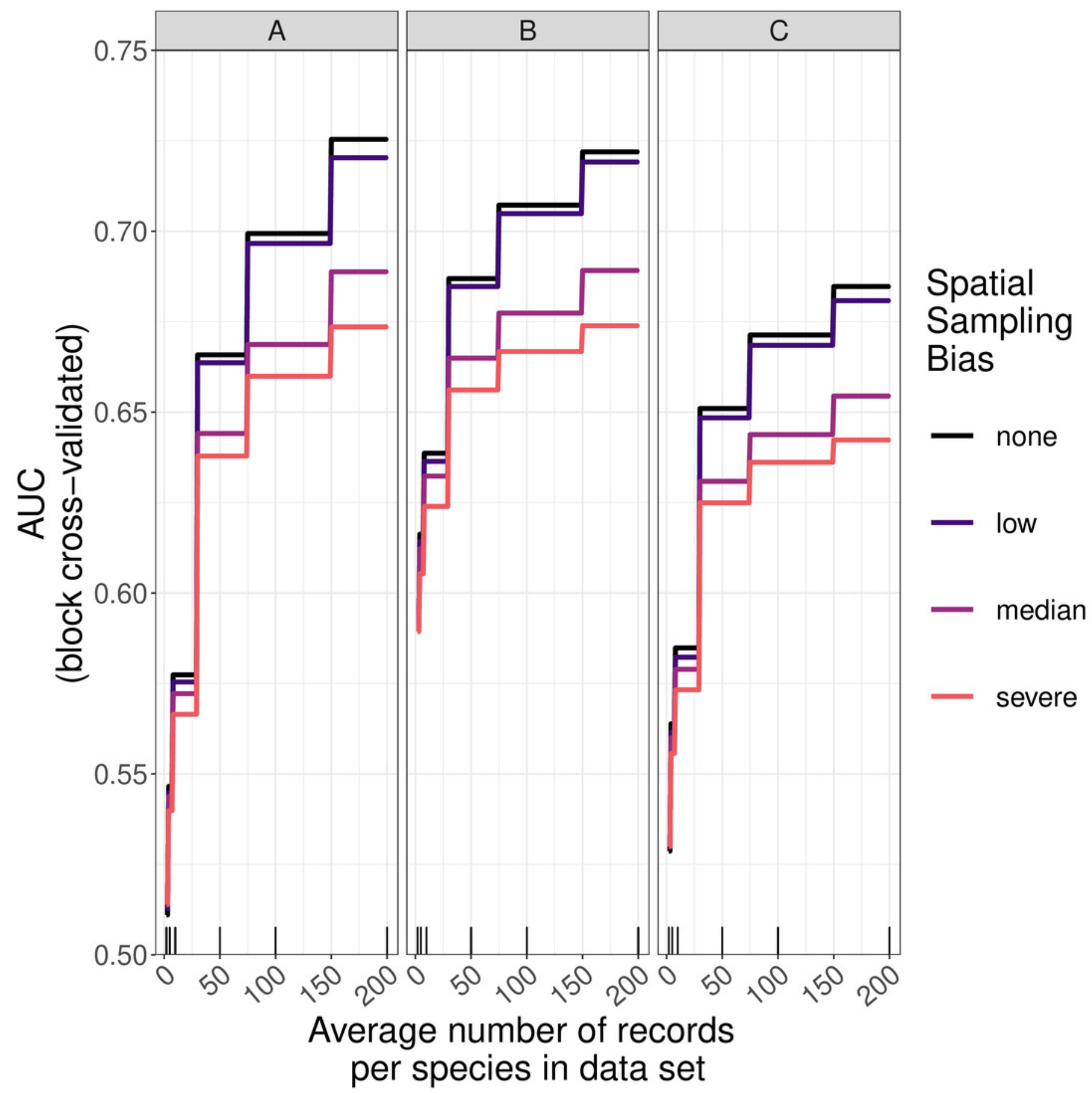




\section{Figure 7}

Observed prediction performance (AUC) of species distribution models for 110 virtual species under a range of sample size and spatial sampling bias scenarios.

Panels show the observed area under the receiver operating characteristic curve (AUC) of species distribution models constructed using (A) generalized linear models, (B) boosted regression trees, and $(\mathrm{C})$ inverse distance-weighted interpolation. Boxes contain the middle $50 \%$ of the observed AUC values. The horizontal line within each box indicates the median AUC value. Each box plot (box, whiskers, and outlying points) represents 110 observations (one for each virtual species) unless models failed to fit for some species (see Fig. 4). The width of boxes is proportional to the square root of the number of observations in that group. 


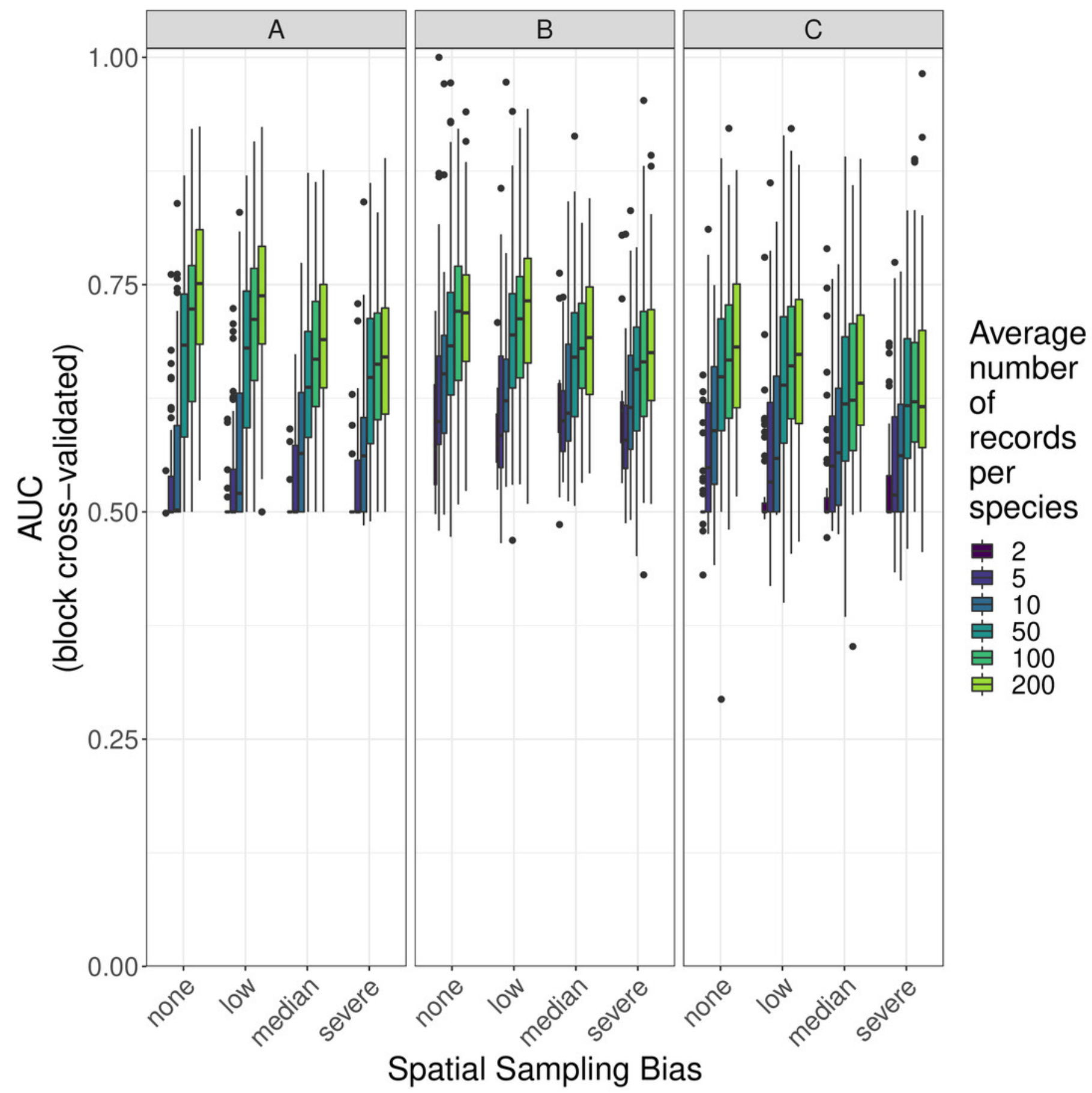




\section{Figure 8}

Observed prediction performance (RMSE) of species distribution models for 110 virtual species under a range of sample size and spatial sampling bias scenarios.

Panels show the observed root mean squared error (RMSE) of species distribution models constructed using (A) generalized linear models, (B) boosted regression trees, and (C) inverse distance-weighted interpolation. Boxes contain the middle $50 \%$ of the observed RMSE values. The horizontal line within each box indicates the median RMSE value. Each box plot (box, whiskers, and outlying points) represents 110 observations (one for each virtual species) unless models failed to fit for some species (see Fig. 4). The width of boxes is proportional to the square root of the number of observations in that group. 


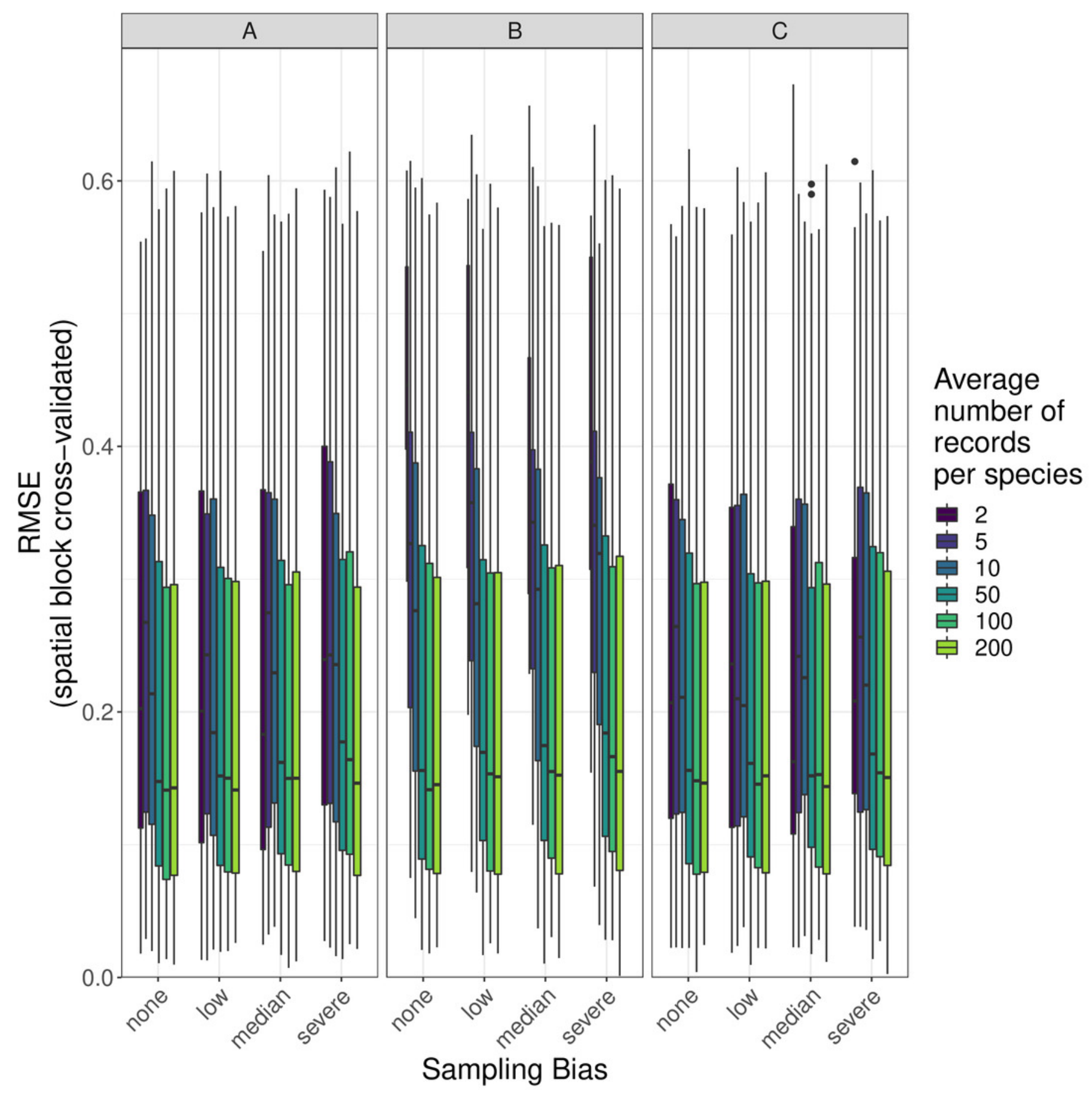




\section{Figure 9}

Contour plot of expected prediction performance of species distribution models as a function of the sample size and spatial sampling bias in virtual biological records datasets.

Expected prediction performance (AUC, contours and shading) of generalized linear model (GLM) species distribution models for virtual species, according to the spatial sampling evenness and sample size of training data. Note the different scales of the horizontal axes in $A$ and $B$. (A) shows detail of prediction performance changes when sample sizes were small. (B) shows larger sample sizes. Spatial sampling evenness was quantified using Simpson evenness. High values of Simpson evenness indicate minimal spatial bias. Open circles show the values of sample size and spatial sampling evenness for virtual biological records datasets used to train species distribution models. Filled black circles show sample size and spatial sampling evenness of Irish biological records datasets used as spatial sampling templates. 

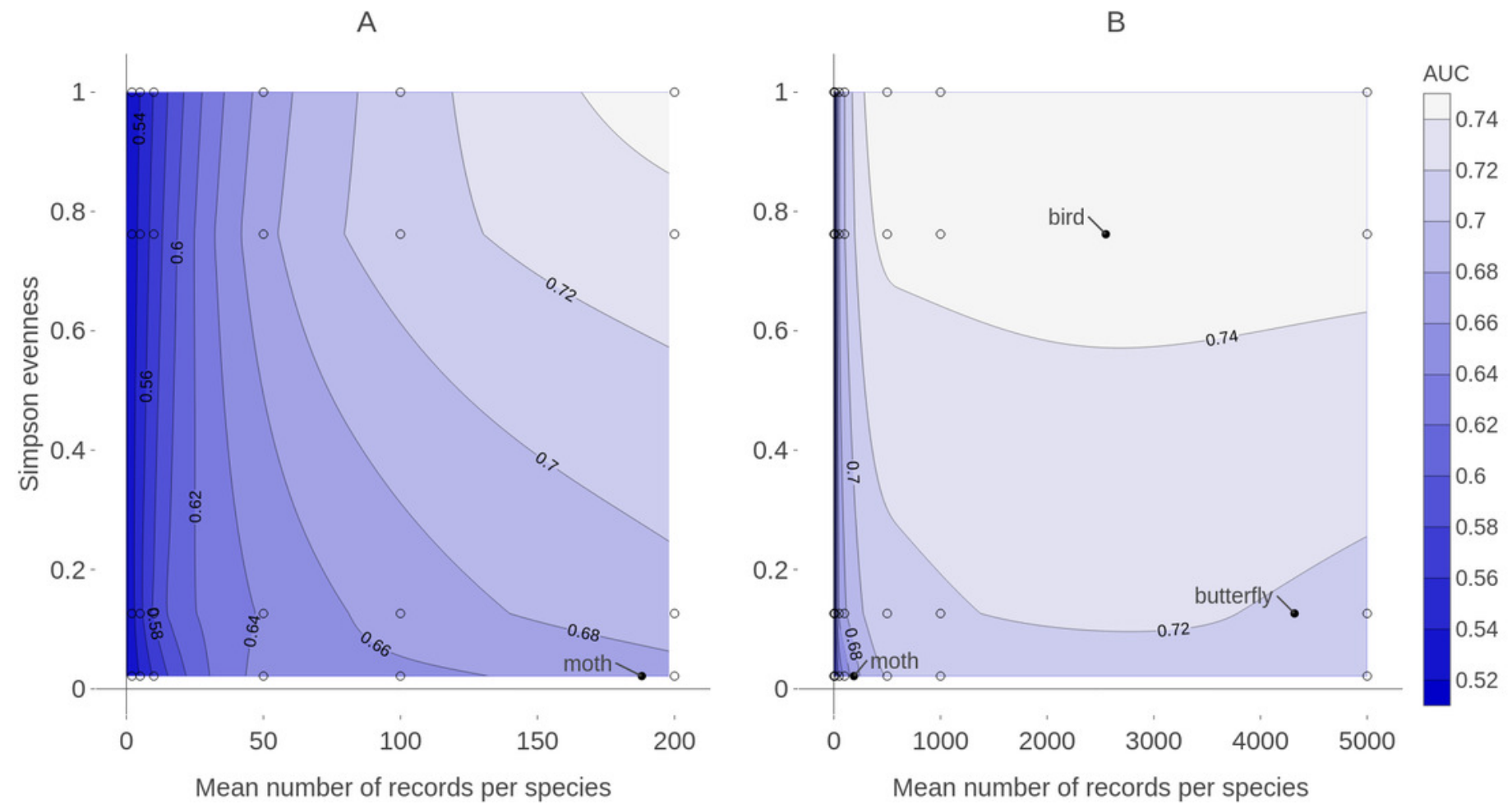


\section{Table $\mathbf{1}$ (on next page)}

Environmental predictor variables used to define and model the distribution of virtual species in Ireland.

Moran's I values indicate the spatial clustering of values for each variable, where a value of one indicates strong spatial clustering of variable values, zero indicates random spatial arrangement of values, and negative one indicates strongly dispersed spatial arrangement of values. Details of data sources are in Section 2.1. 


\begin{tabular}{|c|c|c|c|}
\hline Variable & Description & Data Source & Moran's I \\
\hline $\begin{array}{l}\text { annual minimum } \\
\text { temperature (degrees } \mathrm{C} \text { ) }\end{array}$ & $\begin{array}{l}2 \% \text { quantile of annual temperatures } \\
\text { in each grid cell averaged over the } \\
\text { years } 1995-2016\end{array}$ & E-OBS & 0.84 \\
\hline $\begin{array}{l}\text { annual maximum } \\
\text { temperature (degrees } \mathrm{C} \text { ) }\end{array}$ & $\begin{array}{l}98 \% \text { quantile of annual temperatures } \\
\text { in each grid cell averaged over the } \\
\text { years } 1995-2016\end{array}$ & E-OBS & 0.83 \\
\hline $\begin{array}{l}\text { annual precipitation } \\
(\mathrm{mm})\end{array}$ & $\begin{array}{l}\text { Average total annual precipitation in } \\
\text { each grid cell over the years 1995- } \\
2016 \text { (excluding 2010-2012) }\end{array}$ & E-OBS & 0.82 \\
\hline $\begin{array}{l}\text { average daily sea level } \\
\text { atmospheric pressure } \\
\text { (hecto Pascals) }\end{array}$ & $\begin{array}{l}\text { Average daily sea level atmospheric } \\
\text { pressure over the years } 1995-2016\end{array}$ & E-OBS & 0.86 \\
\hline agricultural areas & $\begin{array}{l}\text { Proportion of each grid cell classified } \\
\text { as agricultural areas }\end{array}$ & $\begin{array}{l}\text { CORINE Land } \\
\text { Cover Database }\end{array}$ & 0.53 \\
\hline artificial surfaces & $\begin{array}{l}\text { Proportion of each grid cell classified } \\
\text { as artificial surfaces }\end{array}$ & $\begin{array}{l}\text { CORINE Land } \\
\text { Cover Database }\end{array}$ & 0.44 \\
\hline $\begin{array}{l}\text { forest and semi-natural } \\
\text { areas }\end{array}$ & $\begin{array}{l}\text { Proportion of each grid cell classified } \\
\text { as forest and semi-natural areas }\end{array}$ & $\begin{array}{l}\text { CORINE Land } \\
\text { Cover Database }\end{array}$ & 0.41 \\
\hline water bodies & $\begin{array}{l}\text { Proportion of each grid cell classified } \\
\text { as water bodies }\end{array}$ & $\begin{array}{l}\text { CORINE Land } \\
\text { Cover Database }\end{array}$ & 0.35 \\
\hline wetlands & $\begin{array}{l}\text { Proportion of each grid cell classified } \\
\text { as wetlands }\end{array}$ & $\begin{array}{l}\text { CORINE Land } \\
\text { Cover Database } \\
\text { ETOPO1 }\end{array}$ & $\begin{array}{l}0.55 \\
029\end{array}$ \\
\hline
\end{tabular}




\section{Table 2 (on next page)}

Spatial sampling evenness of the spatial sampling template datasets measured using Simpson evenness.

A value of one indicates perfectly even sampling (all grid squares containing the same number of records). Lower Simpson evenness values indicate more spatially uneven sampling. 
1

\begin{tabular}{|l|l|}
\hline Spatial sampling template & Simpson evenness value \\
\hline no bias & 1 \\
\hline low bias (birds) & 0.762 \\
\hline median bias (butterflies) & 0.126 \\
\hline severe bias (moths) & 0.021 \\
\hline
\end{tabular}

2 


\section{Table 3 (on next page)}

Importance of sample size, spatial bias, modelling method, and species prevalence for determining predictive performance of species distribution models.

Variable importance measures from a boosted regression tree show the relative influence of sample size (average number of records per species), species distribution modeling method, and spatial bias in training data on three different measures of prediction performance of species distribution models. The relative influence for each variable is the reduction in squared error attributed to that variable in a boosted regression tree model. Prediction performance metrics were area under the receiver operating characteristic curve (AUC), root mean squared error (RMSE) and Cohen's Kappa calculated using the threshold that maximized Kappa (Kappa). The effect of species prevalence was not included for AUC and for Kappa, because exploratory plots showed no indication of an effect. 
2

\begin{tabular}{|l|l|l|}
\hline $\begin{array}{l}\text { Prediction performance } \\
\text { metric }\end{array}$ & Variable & $\begin{array}{l}\text { Relative importance } \\
\text { (reduction in squared } \\
\text { error) }\end{array}$ \\
\hline AUC & Average number of records per species & 78.5 \\
\hline & Species distribution modelling method & 14.8 \\
\hline RMSE & Spatial bias & 6.7 \\
\hline & Species prevalence & 99.9 \\
\hline & Average number of records per species & 0.2 \\
\hline & Species distribution modelling method & 0.003 \\
\hline Kappa & Spatial bias & 0.0001 \\
\hline & Species distribution modelling method & 74.2 \\
\hline & Average number of records per species & 22.4 \\
\hline & Spatial bias & 3.4 \\
\hline
\end{tabular}

